\title{
Outcome of Transjugular Intrahepatic Portosystemic Shunt in Budd-Chiari Syndrome: Long-Term Outcomes of 118 Patients; A Single-center Experience
}

\begin{abstract}
Context: Budd-Chiari syndrome (BCS) is a heterogeneous group of clinical conditions ranging from acute liver failure to completely asymptomatic patient. Patients with BCS nonresponsive to medical treatment or who are not candidates for angioplasty/stenting are routinely treated with transjugular intrahepatic portosystemic shunt (TIPS) to improve portal venous outflow. Aims: This retrospective study aims at determining the short and long-term outcomes of TIPS in BCS patients. Subjects and Methods: This retrospective study included 118 patients with primary BCS who underwent TIPS from 2005 to 2011. They were classified according to Child-Pugh, model for end-stage liver disease (MELD), Rotterdam and BCS-TIPS scores. Short-term outcome of TIPS ( $<1$ year) was determined and included immediate postprocedure complications, effect on clinical status, liver profile, and stent patency. Long-term outcome (1-5 years) was determined and included stent patency rate, time, and pattern of management of stent occlusion and survival rates at 1, 3, and 5 years. Statistical Analysis Used: SPSS statistical package version 16. Paired $t$-test was used to compare pre and post-TIPS MELD and Child-Pugh scores. Chi-square test and Kaplan-Meier survival curve were used to examine the association between short- and long-term occlusion, liver function and survival outcomes. $P<0.05$ was considered statistically significant. Results: By the $1^{\text {st }}$ year, there was a significant decline in the percentage of patients having ascites and abdominal pain, increase in mean serum albumin from 3.42-4.02 $\mathrm{g} / \mathrm{dl}$ and reduction in bilirubin levels from $2.57-1.35 \mathrm{mg} / \mathrm{dl}(P<0.001)$. The rate of stent occlusion by the $1^{\text {st }}$ year was $27.4 \%$. During long-term follow-up, there was a marked improvement in clinical status, liver profile, Child-Pugh, and MELD scores $(P<0.001)$. The 1 - and 5-year shunt patency rates were $72.6 \%$ and $78.7 \%$, respectively. The main management of post-TIPS occlusion was angioplasty without stenting. The 1-, 3-, and 5-year survival rates were $95.8 \%, 93.2 \%$, and $91.5 \%$, respectively. There was a significant relation between survival and post-TIPS MELD score $(P=0.009)$. Conclusions: TIPS is an effective treatment modality for BCS and has a long-term survival benefit with satisfactory shunt patency and patient survival.
\end{abstract}

Keywords: Budd-Chiari syndrome, outcome, stent occlusion, survival, transjugular intrahepatic portosystemic shunt

\section{Introduction}

\section{Budd-Chiari syndrome}

a heterogeneous group conditions ranging from failure to completely asymptomatic patient. It is caused by hepatic venous outflow obstruction at any level from the small hepatic veins (HVs) to the junction of inferior vena cava (IVC) with the right atrium. ${ }^{[1,2]}$ The classic triad of abdominal pain, ascites, and hepatomegaly is commonly present in those patients. ${ }^{[3,4]}$ Ultrasound combined with Doppler imaging has a diagnostic sensitivity of more than $74 \%$ and should be the first line of investigation when the

This is an open access journal, and articles are distributed under the terms of the Creative Commons AttributionNonCommercial-ShareAlike 4.0 License, which allows others to remix, tweak, and build upon the work non-commercially, as long as appropriate credit is given and the new creations are licensed under the identical terms.

For reprints contact: reprints@medknow.com diagnostic features cannot be demonstrated, computed tomography (CT) or preferably magnetic resonance imaging (MRI) should be performed as a second line of investigation. ${ }^{[5]}$

According to Baveno VI consensus workshop, management of BCS should be undertaken using a stepwise approach including anticoagulation, angioplasty/thrombolysis, or transjugular intrahepatic portosystemic shunt (TIPS). ${ }^{[6]}$

Patients with BCS nonresponsive to medical treatment or who are not candidates for angioplasty/stenting may be treated with TIPS to improve portal venous outflow and reduce portal hypertension. TIPS has

How to cite this article: Shaker M, Sakr M, Dabbous H, Abdelhakam SM, Samir A, Ebada H, et al. Outcome of transjugular intrahepatic portosystemic shunt in Budd-Chiari syndrome: Long-term outcomes of 118 patients; A single-center experience. Arab J Intervent Radiol 2018;2:75-81.

\section{Mohamed Shaker, Mohammad Sakr', Hany Dabbous ${ }^{1}$, Sara M. \\ Abdelhakam ${ }^{1}$, Ahmed Samir ${ }^{1}$, Hend Ebada ${ }^{1}$, Amir M. Farid', Mohamed El-Gharib, Ahmed Eldorry}

Departments of Radiodiagnosis and Interventional Radiology and 'Tropical Medicine, Faculty of Medicine, Ain Shams University, Cairo, Egypt

Address for correspondence: Dr. Mohamed Shaker, 13 Mostafa Refaat Street, Sheraton Heliopolis, Cairo, Egypt. E-mail: mohamedshakerghazy@ yahoo.com

Access this article online Website: www.arabjir.com DOI: 10.4103/AJIR.AJIR_11_18 Quick Response Code:

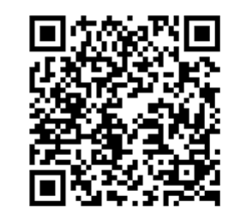


a lower morbidity and mortality rate than surgery and is feasible in most patients with IVC obstruction and those with severe IVC stenosis. ${ }^{[7,8]}$

The aim of the present study was to evaluate the short- and long-term outcomes of TIPS procedure in BCS patients.

\section{Subjects and Methods}

\section{Study design and sampling}

The medical records of 205 primary BCS patients registered by the Budd-Chiari Study Group at a single institution, from December 2005 to January 2011 were revised and analyzed. The Research Ethical Committee approved the study protocol according to the ethical guidelines of the 1975 Declaration of Helsinki. The patterns of management were reported. All patients received anticoagulation therapy as early as possible to reduce the risk of clot extension and new thrombotic episodes. Treatment of the underlying prothrombotic cause was also initiated simultaneously. Acute BCS was diagnosed when a patient developed severe right upper quadrant abdominal pain, hepatomegaly, jaundice, and intractable ascites within few weeks, the subacute form was diagnosed with a more insidious presentation, over several months, while the chronic form was diagnosed when a patient had symptoms or signs for more than 6 months. The compensated liver disease was diagnosed in the absence of signs of decompensation such as jaundice, ascites, variceal hemorrhage, or hepatic encephalopathy (HE).

The inclusion criteria for the current study were as follows: (1) diffuse occlusion of all $\mathrm{HVs}$ with patent portal vein (PV) and IVC which was evaluated by abdominal Duplex ultrasonography (US), multislice computed tomography (CT), abdominal magnetic resonance imaging or (MR) venography; and (2) Cardiac function checked clinically, by ECG and Echocardiography. ${ }^{[9]}$ Exclusion criteria were: (1) Retro- or suprahepatic IVC obstruction; (2) complete PV thrombosis; (3) presence of comorbid etiology for liver disease (e.g. viral hepatitis); (4) hepatocellular carcinoma; (5) cardiac contraindications to TIPS (congestive heart failure and severe pulmonary hypertension); (6) Coagulopathy (INR >2) and thrombocytopenia (platelets $<50000$ ); (7) biliary obstruction; and (8) Uncontrolled sepsis. A cohort of 118 patients met these inclusion criteria and hence were eligible for TIPS procedure and were further analyzed in the current retrospective study. They were 75 females (63.6\%) and 43 males (36.4\%). The mean age at first medical visit was $28.75 \pm 8.55$ years.

\section{Preintervention assessment and preparation}

Baseline laboratory investigations included a complete blood count, liver and renal profile, and coagulation profile. A thrombophilia workup, to determine the underlying etiology of BCS was performed.

Patients were classified according to the following:

a. Modified Child-Pugh score ${ }^{[10]}$ b. Model for end-stage liver disease (MELD) score: $3.8 \times(\mathrm{Ln}$ serum bilirubin $\mathrm{mg} / \mathrm{dL})+11.2 \times(\mathrm{Ln} \mathrm{INR})$ $+9.6 \times($ Ln serum creatinine $\mathrm{mg} / \mathrm{dL})+6.4^{[11]}$

c. Rotterdam prognostic classification into 3 classes according to the equation: $1.27 \times$ encephalopathy +1.04 $\times$ ascites $+0.72 \times$ prothrombin time $+0.004 \times$ bilirubin (Ascites and HE were scored as present (1) or absent (0) and prothrombin time as higher (1) or lower (0) than 2.3 INR. Bilirubin was included as a continuous variable). Where Class I (0-1.1): good prognosis; Class II (1.1-1.5): Intermediate prognosis and Class III $(>1.5)$ : Poor prognosis ${ }^{[2]}$

d. BCS-TIPS score: Age $\times 0.08+$ bilirubin $\times 0.16+$ INR $\times 0.63 .^{[13]}$

These scores were calculated for each patient before starting anticoagulation therapy.

All patients started anticoagulation therapy when the diagnosis of BCS was evident; in the form of low molecular weight heparin (LMWH) or unfractionated heparin. Then oral warfarin was added till INR reached its target (2-3), then continued on oral therapy alone after withdrawal of LMWH or unfractionated heparin. Five days before the procedure, oral anticoagulation therapy was stopped with the administration of LMWH or unfractionated heparin only; to be stopped (6-12 h) before intervention in case of unfractionated heparin and (12-24 h) in case of LMWH. ${ }^{[14]}$

Antibiotic prophylaxis was administered for all patients $(1-2 \mathrm{~h})$ before intervention in the form of a combination of ampicillin-sulbactam $1.5 \mathrm{~g}$ intravenous (IV) and cefotaxime $1 \mathrm{~g} \mathrm{IV} .^{[15]}$

\section{Technical considerations}

TIPS creation was carried out using "Direct Intrahepatic Portocaval Shunt (DIPS) technique." All procedures were done under general anesthesia. The right internal jugular vein was punctured under sonographic guidance followed by insertion of a long 10 French sheath of TIPS set (Cook, USA) over a hydrophilic guidewire 0.035 " (Terumo, Japan) down to the retrohepatic portion of IVC. Using real-time US guidance an $18 \mathrm{G} 20 \mathrm{~cm}$ needle (HS company, Italy) was introduced after percutaneous puncture through intercostal approach into liver parenchyma and advanced till puncturing the right portal branch in an intrahepatic position 1-2 $\mathrm{cm}$ distal to portal bifurcation then the needle is advanced through the liver parenchyma till puncturing the retrohepatic IVC, to create a track between the right portal branch and IVC. A Amplatz super stiff guidewire 0.035" (Boston, USA) was then introduced through the puncture needle so that the tip of the guidewire reached the IVC then snared using a dormia basket (Cook, USA) through the long sheath inserted through the transjugular route and withdrawn till it came out of the sheath at the neck. The puncture needle was withdrawn beyond the right portal branch, and the track between the IVC and 
right portal branch was dilated over the wire by $8 \mathrm{~mm}$ balloon (Boston, USA). The sheath was then advanced down till it reached the right portal branch.

A 5 French catheter (Cobra 2 catheter) (Wanda, Boston, USA) was introduced beside the Super stiff guidewire through the sheath over a hydrophilic guidewire. The catheter was advanced down and manipulated to reach the main PV, and portography was obtained.

The stiff guidewire was then withdrawn out of the sheath and introduced through the Cobra catheter to reach the main PV. The catheter was withdrawn, and then a $10 \mathrm{~mm}$ diameter self-expandable bare metallic stent (Wallstent, Boston Scientific, USA) was introduced over the wire. The long sheath was withdrawn upward till it reached the IVC so that the stent can be deployed and adjusted with lower end at the main PV just below its bifurcation and upper end at the retrohepatic IVC. The stent was dilated using $10 \mathrm{~mm}$ balloon (Wanda, Boston, USA) to get its maximum diameter, and then contrast was injected through the stent to ensure its patency [Figure 1].

The puncture track was embolized through the puncture needle by coil (Cook, USA) to prevent from intraperitoneal bleeding. The pressure gradient was measured between the PV and IVC before and after stent insertion to ensure satisfactory reduction of pressure gradient to $<10 \mathrm{mmHg}$.

\section{Postintervention management}

Patients were admitted to the hospital for 1 week after the procedure for early detection and management of any procedure-related complications and adjustment of

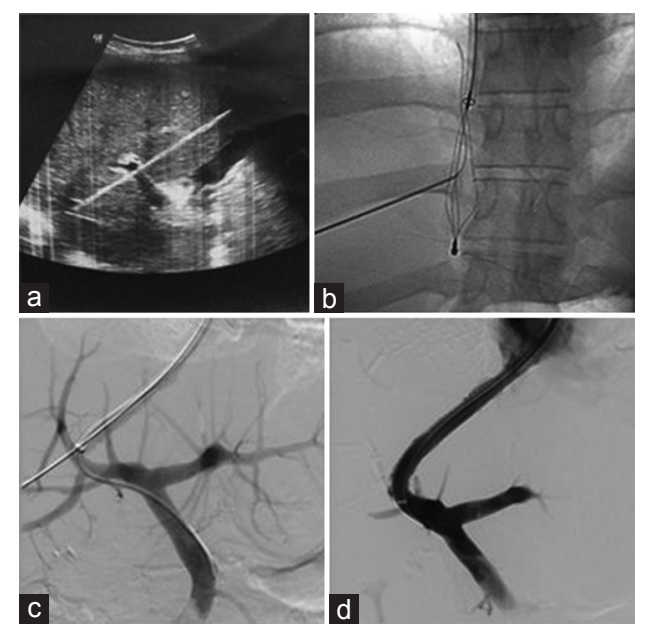

Figure 1: Transjugular intrahepatic portosystemic shunt technique (a) sonography showing puncture needle passing through the right portal branch and reaching retrohepatic inferior vena cava (b) fluoroscopy showing guidewire introduced through the puncture needle and snared by Dormia basket into the long sheath in the retrohepatic inferior vena cava, (c) digital subtraction angiography showing a long sheath inserted to the right portal branch with a manipulation catheter introduced beside the wire into the main portal vein with contrast injection showing portal vein anatomy, and (d) digital subtraction angiography after metallic stent insertion showing contrast flow through the stent from the portal vein to the inferior vena cava anticoagulation therapy. Procedure-related complications were divided into (a) major complications as; hemoperitoneum, biliary peritonitis, stent malposition, hemobilia, radiation skin burn, hepatic infarction, renal failure requiring chronic dialysis, hepatic artery injury, accelerated liver failure, severe or controlled encephalopathy, death (b) minor complications as; transient contrast medium-induced renal failure, encephalopathy controlled by medical therapy, fever, transient pulmonary edema, entry site hematoma. Antibiotics regimen taken before the procedure was continued for 5 days after the procedure. Parenteral anticoagulation (LMWH after $24 \mathrm{~h}$ or unfractionated Heparin after $6 \mathrm{~h}$ ) then oral warfarin therapy was introduced till INR reaches (2-3) then oral therapy was continued alone for life. Duplex US was performed to detect shunt patency immediately after the procedure.

\section{The following follow-up data were retrieved}

1. Short-term outcome ( $<1$ year): In the form of immediate postprocedure complications (intraperitoneal bleeding, HE, or sepsis); effect of procedure on patients' clinical status and liver profile and follow-up of stent patency by Duplex US at days $0,1,3,7,30$, then every 3 months for the $1^{\text {st }}$ year

2. Long-term outcome (1-5 years): Patients were assessed every 3 months for 1 year then every 6 months for the next year then every year or in case of any newly developed relevant symptoms or signs. The assessed items were: (a) Clinical status of the patient, for example, gastrointestinal (GIT) bleeding, ascites, HE. (b) Laboratory investigations mainly liver profile, coagulation profile, and complete blood count. (c) Imaging studies, using duplex US, CT, and/or MR angiography when indicated, to assess stent patency. Patients with occluded stents were subjected to venography (stent revision) followed by angioplasty \pm application of another stent(s) as indicated.

Aims of follow-up were: Assessment of stent patency rate and time of occlusion, pattern of management of stent occlusion (angioplasty with or without stent) and calculation of 1-, 3-, and 5-year survival rates of patients.

\section{Statistical analysis}

Statistical analysis was performed using SPSS statistical package version 16 (IBM, Armonk, NY, USA). Qualitative data were presented as frequency and percentages. Quantitative variables are presented as mean \pm standard deviation, median and range. Paired $t$-test was used to compare pre and post-TIPS MELD and Child-Pugh scores. Chi-square test and Kaplan-Meier survival curve were used to examine the association between short and long-term occlusion, liver function, and survival outcomes. $P<0.05$ was considered statistically significant. 
The statistical methods of this study were reviewed by a statistician.

\section{Results}

The present study included 118 patients with primary BCS who underwent TIPS procedure naively without a preceding intervention. They were 75 females $(63.6 \%)$ and 43 males $(36.4 \%)$. The mean age at first medical visit was $28.75 \pm 8.55$ years.

At first presentation, the mean MELD score was $9.28 \pm 3.8$ and Child-Pugh score was $7.88 \pm 1.62$, where 29 patients $(24.6 \%)$ were Child A, 68 (57.6\%) were Child $\mathrm{B}$ and 21 (17.8\%) were Child C. The mean Rotterdam score of enrolled cohort was $1.3 \pm 0.5$, where 54 patients $(45.8 \%)$ were Class I (good prognosis), 36 (30.5\%) were Class II (intermediate prognosis), and $28(23.7 \%)$ were Class III (poor prognosis). All the enrolled patients had BCS-TIPS score $<7$ with a mean of $3.5 \pm 0.7$, which indicated a good prognostic score.

Among the enrolled cohort, 104 patients (88.1\%) had chronic BCS and 14 (11.9\%) had acute or subacute BCS.

Table 1 summarizes demographic, clinical data, and etiology of studied patients.

The most frequent etiologies were FVLM (44.9\%), and MTHFR mutation (40.7\%).

The etiology of BCS was undefined in eleven patients $(9.3 \%)$. Fifty-six patients $(47.5 \%)$ demonstrated a single etiological factor, 36 (30.5\%) two such factors, $10(8.5 \%)$ three factors, and 5 patients $(4.2 \%)$ had four etiological factors.

Short-term postprocedure complications of transjugular intrahepatic portosystemic shunt $(<1$ year)

Ninety-eight patients $(83 \%)$ had no complications at 30-day, while sepsis occurred in 11 patients $(9.3 \%)$, intraperitoneal hemorrhage in $7(5.9 \%)$, $\mathrm{HE}$ in $2(1.7 \%)$. Thirty-day mortality rate was $4.2 \%$ (5 patients). The cause of death was intraperitoneal hemorrhage in three patients, sepsis in one patient and acute liver failure in one patient.

At 1-year follow-up post-TIPS, there was a significant decline in the percentage of patients having ascites and abdominal pain $(P<0.001)$. There was a statistically significant increase in mean serum albumin and reduction in bilirubin levels following TIPS procedure $(P<0.001)$ [Table 2].

The rate of stent occlusion at 1-year post-TIPS was $27.4 \%$ (31 patients had stent occlusion out of the 113 patients who remained in the study) and patency rate was $72.6 \%(82 / 113)$ [Table 2], where occlusion occurred once in $21 / 31$ patients $(67.7 \%)$ and twice or more in $10 / 31$ patients $(32.3 \%)$. The main management in the 31 patients who had TIPS occlusion was angioplasty
Table 1: Demographic, clinical data, and etiology of studied patients $(n=118)$

\begin{tabular}{lc}
\hline & $\boldsymbol{n} \mathbf{( \% )}$ \\
\hline Age (years), mean \pm SD & $28.75 \pm 8.55$ \\
Gender & \\
Females & $75(63.6)$ \\
Males & $43(36.4)$ \\
Presenting symptoms or signs & \\
Abdominal pain & $106(89.8)$ \\
Upper gastrointestinal bleeding & $15(12.7)$ \\
Ascites & $98(83.1)$ \\
Hepatomegaly & $102(86.4)$ \\
Splenomegaly & $54(45.8)$ \\
Lower limb edema & $39(33.1)$ \\
Jaundice & $24(20.3)$ \\
Hepatic encephalopathy & $11(9.3)$ \\
Etiology & \\
Factor V Leiden mutation & $53(44.9)$ \\
Methylenetetrahydrofolate reductase & $48(40.7)$ \\
Myeloproliferative disorder & $23(19.5)$ \\
Antiphospholipid syndrome & $31(26.3)$ \\
Protein C deficiency & $8(6.8)$ \\
Antithrombin III deficiency & $6(5.1)$ \\
Protein S deficiency & $4(3.4)$ \\
\hline SD: Stangrd
\end{tabular}

SD: Standard deviation

Table 2: Short-term outcome of transjugular intrahepatic portosystemic shunt procedure $(<1$ year) among the 118 Budd-Chiari syndrome patients who underwent transjugular intrahepatic portosystemic

\begin{tabular}{lccc}
\multicolumn{4}{c}{ shunt } \\
\hline & Pre-TIPS & Post-TIPS & $\boldsymbol{P}$ \\
\hline $\begin{array}{l}\text { Impact of TIPS on clinical } \\
\text { status, } n(\%)\end{array}$ & & & \\
$\quad$ Ascites & $98(83.1)$ & $43(36.4)$ & $<0.001$ \\
$\quad$ Abdominal pain & $106(89.8)$ & $55(46.6)$ & $<0.001$ \\
$\quad$ Hepatic encephalopathy & $11(9.3)$ & $9(7.6)$ & 0.810 \\
$\quad$ Upper GIT bleeding & $15(12.7)$ & $13(11)$ & 0.84 \\
Impact of TIPS on liver & & & \\
profile* ${ }^{*}$ mean \pm SD & & & $<0.001$ \\
$\quad$ Albumin & $3.42 \pm 0.69$ & $4.02 \pm 0.43$ & $<0.001$ \\
$\quad$ Bilirubin & $2.57 \pm 2.02$ & $1.35 \pm 0.66$ & \\
Rate of stent occlusion, & & $31 / 113^{* *}(27.4)$ & \\
$n(\%)$ & & & \\
Patency rate, $n(\%)$ & & $82 / 113(72.6)$ & \\
\hline
\end{tabular}

*INR was not studied because of the effect of anticoagulation, ${ }^{* *} 5$ patients died in short term follow-up. SD: Standard deviation, TIPS: Transjugular intrahepatic portosystemic shunt, GIT: Gastrointestinal tract

without stenting in $25 / 31$ patients $(80.6 \%)$ and angioplasty with stenting in $6 / 31$ patients $(19.4 \%)$.

Long-term outcome of transjugular intrahepatic portosystemic shunt procedure (1-5 years)

There was marked improvement in the survived patients where $86.7 \%$ (98/113) had compensated liver disease, 
and there was a statistically significant increase in mean serum albumin and reduction in bilirubin levels. Both Child-Pugh and MELD scores were significantly improved post-TIPS $(P<0.001)$ [Table 3$]$.

During the long-term follow-up, five patients died ( 2 from upper GIT bleeding, 2 from liver cell failure, and 1 from sepsis) and 108 patients continued the follow-up. The rate of stent occlusion was 23/108 (21.3\%) and 5-year patency rate was $78.7 \%(85 / 108)$ [Table 3 ], $(11 / 23$ patients $(47.8 \%)$ were occluded once, and $12 / 23$ patients $(52.2 \%)$ had occlusion twice or more, where $11 / 108$ patients $(10.2 \%)$ developed stent occlusion during the $2^{\text {nd }}$ year, $4 / 108(3.7 \%)$ during the $3^{\text {rd }}$ year, $5 / 108(4.6 \%)$ during the $4^{\text {th }}$ year, and $3 / 108$ patients $(2.8 \%)$ had their occlusion during the $5^{\text {th }}$ year of follow-up. Out of these 23 patients, 10 cases were occluded during the $1^{\text {st }}$ year and occluded again after 1 year.

The main management post-TIPS occlusion was angioplasty without stent in $18 / 23$ patients $(78.3 \%)$ and angioplasty with the stent in 5/23 (21.7\%).

The 1-, 3-, and 5-year orthotopic liver transplantation (OLT)-free survival rates of studied patients were $95.8 \%, 93.2 \%$, and $91.5 \%$, respectively. Figure 2 shows Kaplan-Meier curve for the overall survival following TIPS procedure.

Regarding the relation between post-TIPS 5-year survival and different prognostic scores, the post-TIPS MELD was the only score that showed significant correlation with patients' survival $(P=0.009)$ [Table 4].

\section{Discussion}

According to recently published data, TIPS should be indicated in the management of BCS if angioplasty is not technically feasible or in cases of severe portal hypertension. ${ }^{[7,16,17]}$

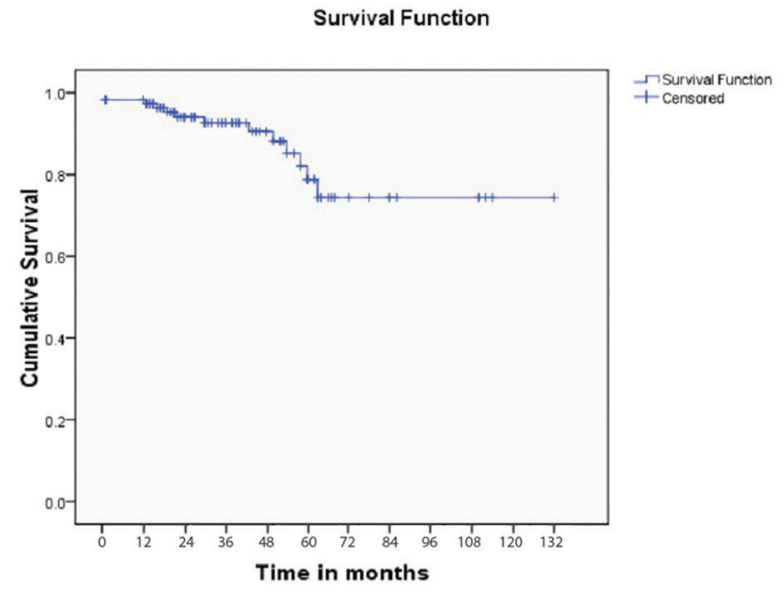

Figure 2: Kaplan-Meier curve for overall survival following transjugular intrahepatic portosystemic shunt procedure. The 1-, 3-, and 5-year survival rates were $95.8 \%, 93.2 \%$, and $91.5 \%$, respectively
The current retrospective study included 118 patients with primary BCS who had diffuse occlusion of the three HVs with patent PV and IVC. Therefore, all were eligible for TIPS technique.

Most of the enrolled patients had an intermediate severity of liver dysfunction with good or intermediate prognosis at presentation. Child B class represented $57.6 \%$ of the recruited patients with mean Child, MELD, and BCS-TIPS scores of $7.88 \pm 1.62,9.28 \pm 3.8$, and $3.5 \pm 0.7$, respectively. According to Rotterdam score, patients were distributed as Class I (45.8\%), Class II (30.5\%), and Class III (23.7\%).

In the current study, sepsis occurred in $9.3 \%$ of cases In spite prophylactic antibiotics, a rate that is higher than what

\begin{tabular}{|c|c|c|c|}
\hline & Pre-TIPS & Post-TIPS & $P$ \\
\hline \multicolumn{4}{|l|}{$\begin{array}{l}\text { Impact on clinical status, } \\
n(\%)\end{array}$} \\
\hline Compensated liver disease & $20(17.7)$ & $98(86.7)$ & $<0.001$ \\
\hline Hepatic encephalopathy & $11(9.7)$ & $5(4.4)$ & $<0.001$ \\
\hline Upper GIT bleeding & $15(13.3)$ & $2(1.8)$ & $<0.001$ \\
\hline \multicolumn{4}{|l|}{$\begin{array}{l}\text { Impact on liver profile, } \\
\text { mean } \pm \text { SD }\end{array}$} \\
\hline Albumin & $3.42 \pm 0.69$ & $4.12 \pm 0.41$ & $<0.001$ \\
\hline Bilirubin & $2.57 \pm 2.02$ & $1.20 \pm 0.62$ & 0.0143 \\
\hline \multicolumn{4}{|l|}{$\begin{array}{l}\text { Impact on Child and MELD } \\
\text { scores, mean } \pm \text { SD }\end{array}$} \\
\hline Child-Pugh & $7.88 \pm 1.62$ & $5.46 \pm 0.69$ & $<0.001$ \\
\hline MELD & $9.28 \pm 3.84$ & $4.56 \pm 1.44$ & $<0.001$ \\
\hline $\begin{array}{l}\text { Rate of stent occlusion*, } \\
n(\%)\end{array}$ & & $23 / 108 * *(21.3)$ & \\
\hline Patency rate, $n(\%)$ & & $85 / 108(78.7)$ & \\
\hline
\end{tabular}

*10 patients were occluded in early and re-occluded in long-term follow-up, $* * 5$ patients died in long-term follow-up. TIPS: Transjugular intrahepatic portosystemic shunt, SD: Standard deviation, MELD: Model for end-stage liver disease, GIT: Gastrointestinal tract

Table 4: Relation between post-transjugular intrahepatic portosystemic shunt survival and different prognostic scores (mean \pm standard deviation)

\begin{tabular}{lccc}
\hline & Survivors & Non- survivors & $\boldsymbol{P}$ \\
\hline Child score & & & \\
Pre-TIPS & $7.66 \pm 1.54$ & $7.88 \pm 1.62$ & 0.312 \\
Post-TIPS & $5.39 \pm 0.67$ & $5.46 \pm 0.69$ & 0.455 \\
MELD score & & & \\
$\quad$ Pre-TIPS & $9.21 \pm 4.02$ & $9.28 \pm 3.48$ & 0.891 \\
$\quad$ Post-TIPS & $4.06 \pm 1.33$ & $4.56 \pm 1.44$ & 0.009 \\
Rotterdam score & & & \\
$\quad$ Pre-TIPS & $1.331 \pm 0.385$ & $1.436 \pm 0.651$ & 0.316 \\
BCS-TIPS score & & & \\
$\quad$ Pre-TIPS & $3.576 \pm 0.931$ & $3.428 \pm 0.804$ & 0.492 \\
\hline
\end{tabular}

TIPS: Transjugular intrahepatic portosystemic shunt, MELD: Model for end-stage liver disease, BCS-TIPS score: Budd-Chiari syndrome-TIPS score 
was recently reported by Tripathi et al., ${ }^{[16]}$ who reported a rate of post-TIPS sepsis of $5 \%$. Whether or not prophylactic antibiotics are of value, remains undetermined. ${ }^{[15,17,18]}$

In our study, all patients started anticoagulation therapy when the diagnosis of BCS was evident. Five days before the procedure, oral anticoagulation therapy was stopped with the administration of LMWH or unfractionated heparin only; to be stopped (6-12 h) before intervention in case of unfractionated heparin and (12-24 h) in case of LMWH.

In the current study, postprocedure bleeding was encountered in seven patients (5.9\%); all were intraperitoneal. Unfortunately, three cases died, in spite, resuscitation measures and withholding anticoagulation. These patients deteriorated rapidly and were not eligible for surgical intervention. The remaining four patients were rescued by surgical intervention. This might be explained by the evident hepatic congestion; the technical difficulty that might dictate repeated hepatic parenchymal punctures as well as the anticoagulation therapy. In fact, a TIPS procedure is technically more challenging in a patient with BCS as compared to cirrhosis. ${ }^{[19]}$

Post-TIPS HE occurred in $1.7 \%$ of the current series. This is not consistent with Tripathi et al., ${ }^{[20]}$ who reported a higher incidence of HE post-TIPS (17\%). The relatively preserved hepatic parenchyma in BCS patients might explain the low rate of HE following TIPS as the underlying hepatic pathology is mainly congestion precipitating fibrosis rather than cirrhosis (especially in early disease stages). ${ }^{[21]}$

Regarding the short-term outcome following TIPS technique, it is worth mentioning that $83 \%$ of the current series had no complications. The 30 days mortality rate was $4.2 \%$. In previous reports, the 30 days mortality post-TIPS varied from $0 \%$ to $36 \%$. The seeming heterogeneity among studies is attributed to the severity of hepatic venous occlusion, degree of liver dysfunction, and inclusion criteria (e.g., malignancy and other concomitant diseases). ${ }^{[22]}$

In regards to the short-term impact of TIPS on the clinical status, the most significant clinical change was the dramatic improvement of ascites during the first few weeks after TIPS. Other symptoms and signs took more time to improve such as abdominal pain, tenderness, HE and GIT bleeding.

Regarding the long-term outcomes, most of the cases (86.7\%) had compensated liver, but some complications occurred especially in patients with occluded stents such as HE (4.4\%), upper GIT bleeding (1.8\%), and mortality rate was $4.4 \%$. These results were inferior to that found by Zahn et al., ${ }^{[23]}$ who reported that none of the patients developed clinically relevant $\mathrm{HE}$ although 11 of 13 cases $(84.6 \%)$ have developed shunt dysfunction.
Five-year shunt patency was 78.7\%. Twenty-three patients $(21.3 \%)$ needed re-intervention; 11 for once and 12 twice or more. This compares favorably to what was reported by Garcia-Pagán et al., ${ }^{[13]}$ where $49 \%$ had TIPS dysfunction in a series of 124 patients during the follow-up. It worth mentioning that Tripathi et al. found that primary patency rates at 5 years for covered stents was $70 \%$ versus $27 \%$ for bare stents.

Regarding the impact of TIPS procedure on Child-Pugh and MELD scores, both scores significantly improved following TIPS. This was consistent with Qi et al. ${ }^{[22]}$ However, calculating MELD score in patients with chronic BCS may be problematic because many of these patients are on anticoagulation. In the current study, the improvement in other 2 parameters of MELD (bilirubin and creatinine) was more noticeable that it could overcome the effect of anticoagulation on INR results.

In the current study, the 1-, 3-, and 5-year OLT-free survival rates were $95.8 \%, 93.2 \%$, and $91.5 \%$, respectively. Due to the rarity of $\mathrm{BCS}$, reports on survival after TIPSS are limited. In 2008, a large European multicenter retrospective study reported 12- and 60-month OLT-free survival of $88 \%$ and $78 \% \cdot{ }^{[13]}$ In the retrospective study of Tripathi et al., ${ }^{[16]}$ the reported survival rates following TIPSS were $92 \%$, $80 \%$, and $72 \%$ at 12,60 , and 120 months, respectively.

We found that the Child-Pugh and pre-TIPS MELD scores had no significant association with survival. However, the post-TIPS MELD score showed significant association with survival. Our observations are inconsistent with Khuroo et al. ${ }^{[24]}$ who stated that the Child-Pugh score was independently predictive of survival.

\section{Conclusions}

TIPS creation is an effective treatment for eligible BCS patients and has long-term survival benefit with satisfactory shunt patency and patient survival.

Financial support and sponsorship

Nil.

Conflicts of interest

There are no conflicts of interest.

\section{References}

1. DeLeve LD, Valla DC, Garcia-Tsao G; American Association for the Study Liver Diseases. Vascular disorders of the liver. Hepatology 2009;49:1729-64.

2. Martens P, Nevens F. Budd-Chiari syndrome. United European Gastroenterol J 2015;3:489-500.

3. Darwish Murad S, Plessier A, Hernandez-Guerra M, Fabris F, Eapen CE, Bahr MJ, et al. Etiology, management, and outcome of the Budd-Chiari syndrome. Ann Intern Med 2009;151:167-75.

4. Hadengue A, Poliquin M, Vilgrain V, Belghiti J, Degott C, Erlinger S, et al. The changing scene of hepatic vein thrombosis: Recognition of asymptomatic cases. Gastroenterology 1994;106:1042-7. 
5. Millener P, Grant EG, Rose S, Duerinckx A, Schiller VL, Tessler FN, et al. Color Doppler imaging findings in patients with Budd-Chiari syndrome: Correlation with venographic findings. AJR Am J Roentgenol 1993;161:307-12.

6. de Franchis R; Baveno VI Faculty. Expanding consensus in portal hypertension: Report of the baveno VI consensus workshop: Stratifying risk and individualizing care for portal hypertension. J Hepatol 2015;63:743-52.

7. European Association for the Study of the Liver. Electronic address: easloffice@easloffice.eu. EASL clinical practice guidelines: Vascular diseases of the liver. J Hepatol 2016;64:179-202.

8. Fitsiori K, Tsitskari M, Kelekis A, Filippiadis D, Triantafyllou K, Brountzos E, et al. Transjugular intrahepatic portosystemic shunt for the treatment of Budd-Chiari syndrome patients: Results from a single center. Cardiovasc Intervent Radiol 2014;37:691-7.

9. Cura M, Haskal Z, Lopera J. Diagnostic and interventional radiology for Budd-Chiari syndrome. Radiographics 2009;29:669-81.

10. Pugh RN, Murray-Lyon IM, Dawson JL, Pietroni MC, Williams R. Transection of the oesophagus for bleeding oesophageal varices. Br J Surg 1973;60:646-9.

11. Wiesner R, Edwards E, Freeman R, Harper A, Kim R, Kamath P, et al. Model for end-stage liver disease (MELD) and allocation of donor livers. Gastroenterology 2003;124:91-6.

12. Darwish Murad S, Valla DC, de Groen PC, Zeitoun G, Hopmans JA, Haagsma EB, et al. Determinants of survival and the effect of portosystemic shunting in patients with Budd-Chiari syndrome. Hepatology 2004;39:500-8.

13. Garcia-Pagán JC, Heydtmann M, Raffa S, Plessier A, Murad S, Fabris F, et al. TIPS for Budd-Chiari syndrome: Long-term results and prognostics factors in 124 patients. Gastroenterology 2008;135:808-15.

14. Madura JA, Rookstool M, Wease G. The management of patients on chronic Coumadin therapy undergoing subsequent surgical procedures. Am Surg 1994;60:542-6.
15. Ryan JM, Ryan BM, Smith TP. Antibiotic prophylaxis in interventional radiology. J Vasc Interv Radiol 2004;15:547-56.

16. Tripathi D, Macnicholas R, Kothari C, Sunderraj L, Al-Hilou H, Rangarajan $\mathrm{B}$, et al. Good clinical outcomes following transjugular intrahepatic portosystemic stent-shunts in Budd-Chiari syndrome. Aliment Pharmacol Ther 2014;39:864-72.

17. Peynircioglu B, Shorbagi AI, Balli O, Cil B, Balkanci F, Bayraktar $\mathrm{Y}$, et al. Is there an alternative to TIPS? Ultrasound-guided direct intrahepatic portosystemic shunt placement in Budd-Chiari syndrome. Saudi J Gastroenterol 2010;16:315-8.

18. Beddy P, Ryan JM. Antibiotic prophylaxis in interventional radiology - Anything new? Tech Vasc Interv Radiol 2006;9:69-76.

19. Terreni N, Vangeli M, Raimondo ML, Tibballs JM, Patch D, Burroughs AK, et al. Late intrahepatic hematoma complicating transjugular intrahepatic portosystemic shunt for Budd-Chiari syndrome. Cardiovasc Intervent Radiol 2007;30:1065-9.

20. Tripathi D, Sunderraj L, Vemala V, Mehrzad H, Zia Z, Mangat $\mathrm{K}$, et al. Long-term outcomes following percutaneous hepatic vein recanalization for Budd-Chiari syndrome. Liver Int 2017;37:111-20.

21. Aydinli M, Bayraktar Y. Budd-Chiari syndrome: Etiology, pathogenesis and diagnosis. World J Gastroenterol 2007;13:2693-6.

22. Qi X, Yang M, Fan D, Han G. Transjugular intrahepatic portosystemic shunt in the treatment of Budd-Chiari syndrome: A critical review of literatures. Scand J Gastroenterol 2013;48:771-84.

23. Zahn A, Gotthardt D, Weiss KH, Richter G, Schmidt J, Stremmel W, et al. Budd-Chiari syndrome: Long term success via hepatic decompression using transjugular intrahepatic porto-systemic shunt. BMC Gastroenterol 2010;10:25.

24. Khuroo MS, Al-Suhabani H, Al-Sebayel M, Al Ashgar H, Dahab S, Khan MQ, et al. Budd-Chiari syndrome: Long-term effect on outcome with transjugular intrahepatic portosystemic shunt. J Gastroenterol Hepatol 2005;20:1494-502. 